\title{
Diagnostic Laparoscopy and Removal of Migrated Orthopedic Hardware
}

\author{
John T. English III, MD, Kurt R. Stahlfeld, MD, Cheryl Six, MD \\ Department of Surgery, University of Pittsburgh Medical Center, Pittsburgh, Pennsylvania, USA (all authors).
}

\begin{abstract}
Introduction: Laparoscopic retrieval of orthopedic hardware is rarely documented. We report a case of a diagnostic laparoscopy and successful laparoscopic removal of a migrated retroperitoneal pelvic screw with subsequent resolution of the patient's pain.

Case Description: A 43-year-old female pedestrian was admitted after being struck by a motor vehicle. Included in her many orthopedic injuries were fractures of the right iliac wing and acetabulum necessitating open reduction and internal fixation using an anterior ilioinguinal approach. Four months after her accident, she presented to the outpatient surgery clinic complaining of pain in the right lower quadrant and a protrusion near the ilioinguinal incision. A plain abdominal radiograph revealed that one of the screws used in pelvic fixation had migrated. A diagnostic laparoscopy to evaluate the protrusion noted laxity without hernia at the iliac crest and a firm mass in the retroperitoneum, consistent with orthopedic hardware. The retroperitoneum was incised and the migrated screw was identified, grasped with a laparoscopic grasper, unscrewed from the pelvis, and removed. She was discharged that day and was pain free on follow-up visits.
\end{abstract}

Conclusion: The many benefits of laparoscopy in evaluating the peritoneal cavity can safely be extended to removal of orthopedic hardware.

Key Words: Foreign body, Laparoscopy, Orthopedics.

Citation English JT, Stahlfeld KR, Six C. Diagnostic laparoscopy and removal of migrated orthopedic hardware. CRSLS e2014.00171. DOI: 10.4293/CRSLS.2014.00171.

Copyright (C) 2014 SLS This is an open-access article distributed under the terms of the Creative Commons Attribution-Noncommercial-ShareAlike 3.0 Unported license, which permits unrestricted noncommercial use, distribution, and reproduction in any medium, provided the original author and source are credited.

Address correspondence to: John T. English III, MD, UPMC, 1400 Locust St, Pittsburgh PA 15219, USA. Telephone: 412-232-5528, Fax: 412-233-8096, E- mail: englishjt@upmc.edu

\section{INTRODUCTION}

The benefits of laparoscopic surgery are incontrovertible, and the available equipment and technology are expanding rapidly. General surgeons continue to push the envelope for indications and use of laparoscopy, and thoracic and urologic surgeons are developing laparoscopic skills and procedures for their specialties.

Although orthopedic surgeons are familiar with arthroscopy, laparoscopy has limited use in orthopedics and is primarily restricted to general surgery, particularly for exposure in spinal operations. Because the peritoneal cavity is not in the orthopedic comfort zone, general surgeons with laparoscopic skills may discover another avenue for expanding the use of laparoscopy. In our case, we avoided making an additional incision through already lax fascia and were able to remove a migrated screw via a 5-mm incision.

\section{CASE DESCRIPTION}

A 43-year-old woman involved in a pedestrian versus motor vehicle accident presented with right-sided fractures of the humerus, styloid process, tibia, fibula, iliac wing, acetabulum, medial malleolus, and multiple lumbar, thoracic, and cervical vertebrae. In addition to multiple orthopedic procedures, the iliac and acetabular fracture required open reduction and internal fixation through an anterior ilioinguinal approach. She was discharged to a rehabilitation facility 17 days after the initial injury.

Four months later, the patient presented to the general surgery clinic with complaints of pain and a protrusion 

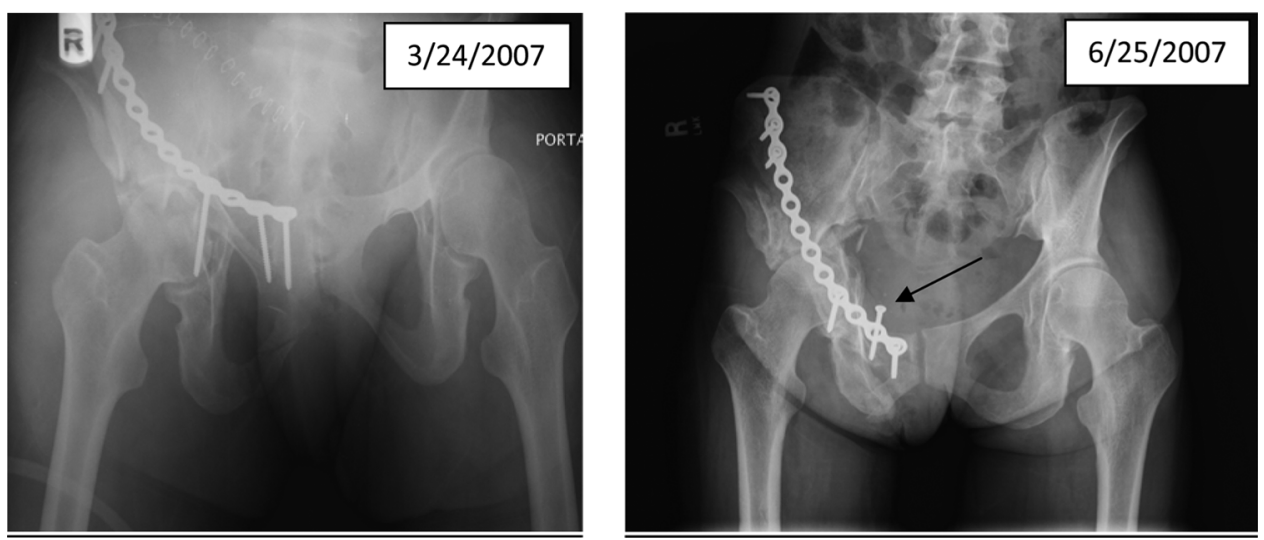

Figure 1. Left: position of the screws after the initial operation. Right: position of the screws showing the migrated screw before the second operation (arrow).

on the right side in the area of the ilioinguinal incision. Physical examination findings were consistent with an incisional hernia or postoperative denervation with muscular atrophy immediately superior to the iliac crest. A follow-up abdominal radiograph ordered by orthopedics was compared to prior imaging and clearly revealed that one of the screws used in the pelvic repair had migrated (Figure 1). Rather than undergo open reexploration, the patient consented to a diagnostic laparoscopy to evaluate the protrusion and for possible herniorrhaphy or hardware removal. At laparoscopy, eventration without fascial defect was noted at the site of the prior incision, and a well-defined, small, firm mass was seen in the retroperitoneum (Figure 2). The

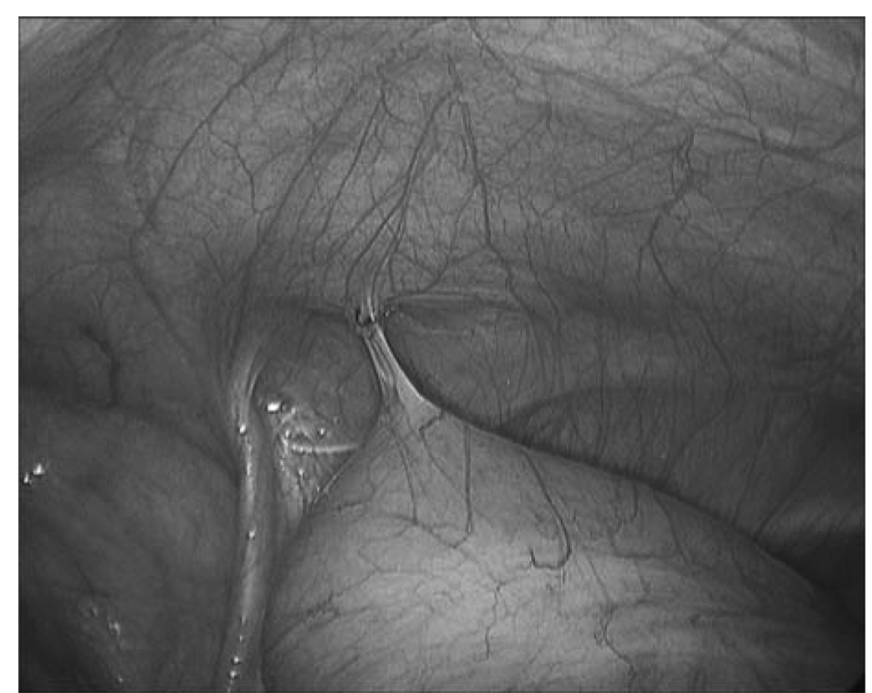

Figure 2. Photograph showing the head of the migrated screw. peritoneum overlying the mass was incised, and the head of the loose pelvic screw was identified, secured with a laparoscopic grasper, unscrewed from the pelvis, and withdrawn through the umbilical port. No treatment was necessary for the eventration; total operative time was 20 minutes.

The patient was discharged home that day and her pain resolved.

\section{DISCUSSION}

Excluding two cases of pin migration, transperitoneal laparoscopic removal of orthopedic hardware has not been reported in the English literature. The etiology of migration is enigmatic and may be related to muscle movement, mechanical stresses, local resorption of bone, and varying pressures in the different body cavities. Confounding any further scientific study is the infrequency of hardware placement near the peritoneal cavity and the mindset that the best route for retrieving the hardware is the route of initial access. Moreover, if a surgeon familiar with minimally invasive techniques is not involved initially, the laparoscopic approach is not considered at the time of the second operation.

Our patient was treated by the trauma service and cared for by orthopedic surgeons and general surgeons familiar with current laparoscopic techniques. The laparoscopic approach proposed by the general surgeons provided this patient with the usual benefits of reduced postoperative pain, improved cosmesis, and reduced hospital stay. It also reduced the risk of infection, which 
would have been magnified by making an incision through a previous scar and exposing the previously placed pelvic plate. However, the greatest advantage was being able to identify the protrusion as an eventration and not a fascial defect, avoiding another incision through already attenuated tissue.

\section{CONCLUSION}

Laparoscopic retrieval of migrated orthopedic hardware is practical, technically feasible, and beneficial to the patient.

\section{Reference:}

1. Potter FA, Fiorini AJ, Knox J, Rajesh PB. The migration of a Kirschner wire from shoulder to spleen: brief report. Bone Joint Surg Br. 1988:70(2):326-327.

2. Antonacci AC, Rosser J. The laparoscopic retrieval of an orthopedic fixation pin from the liver with repair of an associated diaphragmatic laceration. JSLS 2001;5(2):191-195. 